\title{
THE EMBEDDING OF HOMEOMORPHISMS IN FLOWS
}

\author{
M. K. FORT, JR. ${ }^{1}$
}

1. Introduction. Let $X$ be a topological space, and let $R$ be the real number system. If $F$ is a function on $X \times R, x \in X$ and $t \in R$, we will usually denote $F(x, t)$ by $F_{t}(x)$. For each real number $t, F_{t}$ denotes the obvious function on $X$.

A (topological) flow on $X$ is a continuous function $F$ on $X \times R$ into $X$ such that:

(1) $F_{t}$ is a homeomorphism of $X$ onto $X$ for each $t \in R$; and

(2) $F_{t}\left(F_{s}(x)\right)=F_{t+s}(x)$ for all $t \in R, s \in R$ and $x \in X$.

We now state the general embedding problem for flows.

Embedding Problem. For a given space $X$ and a given homeomorphism $f$ of $X$ onto $X$, does there exist a flow $F$ on $X$ for which $F_{1}=f$ ?

If such a flow $F$ exists, we say that $f$ is embedded in $F$.

In general, the embedding problem is quite difficult. In this paper we discuss only the case in which $X$ is an interval of real numbers.

If $X$ is an interval of real numbers and $f$ is a continuously differentiable homeomorphism of $X$ onto $X$, we may ask whether or not $f$ can be embedded in a flow $F$ for which each homeomorphism $F_{t}$ has a continuous derivative. We obtain some results pertaining to the solution of this latter problem, although a complete solution is not obtained.

2. The embedding problem for intervals. Let $f$ be a homeomorphism of an interval of real numbers onto itself. In order that it be possible to embed $f$ in a flow, it is obviously necessary that $f$ be order preserving. We prove that this condition is also sufficient.

LEMMA 1. If $h$ is a homeomorphism of a closed interval $[a, b]$ onto itself such that $a$ and $b$ are the only fixed points of $h$, then it is possible to embed $h$ in a flow $H$ such that if $a<x<b$ and $-1<t<1$ then $H_{t}(x)$ is between $h^{-1}(x)$ and $h(x)$.

Proof. It is proved in [1] and [2] that there exists an order preserving homeomorphism $\psi$ on $[a, b]$ onto $[0, \infty]$ and a positive number $A$ such that $h(x)=\psi^{-1}(A \psi(x))$ for $a \leqq x \leqq b$. If $f(x)>x$ for $a<x<b$,

Presented to the Society, November 27, 1954; received by the editors February 14, 1955.

1 This research has been supported by National Science Foundation grant NSFG1353. 
then $A>1$; if $f(x)<x$ for $a<x<b$, then $A<1$. If we now define $H_{t}(x)=\psi^{-1}(A \psi(x))$ for $a \leqq x \leqq b$ and $t \in R$, then it is easy to verify that $H$ has the desired properties.

THEOREM 1. If $f$ is an order preserving homeomorphism of an interval $J$ onto itself, then it is possible to embed $f$ in a flow $F$.

Proof. We may assume without loss of generality that $J$ is a closed interval. Let $K$ be the set of all fixed points of $f$, and let $\Sigma$ be the set of all closed intervals which are the closures of the components of $J-K$. For each interval $u \in \Sigma$, we use Lemma 1 to obtain a flow $U$ on $u$ such that $U_{1}=f \mid u$, and such that for $-1<t<1$ and each point $x$ that is interior to $U, u_{t}(x)$ is between $f^{-1}(x)$ and $f(x)$.

Now let $x$ be a member of $J$. If $x \in K$, we define $F_{t}(x)=x$ for all $t \in R$. If $x \in J-K$, then there exists $u \in \Sigma$ such that $x \in u$; and in this case we define $F_{t}(x)=U_{t}(x)$ for all $t \in R$.

For each $t, F_{t}$ is a one-to-one order preserving function on $J$ onto $J$, and it follows that $F_{t}$ is a homeomorphism. Moreover, if $t \in R, s \in R$ and $x \in J$, then it is easy to verify that $F_{t}\left(F_{s}(x)\right)=F_{t+s}(x)$.

We must finally prove that $F$ is continuous on $J \times R$. Since each $F_{t}$ is continuous and $F_{t} F_{s}=F_{t+s}$ for all $t, s$, it is sufficient to prove that $F$ is continuous at each point of the form (a,0), $a \in J$. If $a \in J-K$, it is obvious that $F$ is continuous at $(a, 0)$. Thus, let us assume that $a \in K$ and that $\left(x_{n}, t_{n}\right) \rightarrow(a, 0)$ as $n \rightarrow \infty$. For all large values of $n$ we have $-1<t_{n}<1$, and hence $F\left(x_{n}, t_{n}\right)$ is between $f^{-1}\left(x_{n}\right)$ and $f\left(x_{n}\right)$. Since $f$ and $f^{-1}$ are continuous and $f(a)=f^{-1}(a)=F(a, 0)$, it follows that $F\left(x_{n}, t_{n}\right) \rightarrow F(a, 0)$ as $n \rightarrow \infty$. Thus $F$ is continuous.

If the function $h$ of the lemma has a continuous derivative, then the function $\psi$ may be chosen so as to have a continuous derivative on the open interval $(a, b)$. It follows that each function $H_{t}$ has a continuous derivative on the open interval $(a, b)$. Turning now to the above theorem, we see that if $f$ has a continuous derivative on $J$, then the flow $F$ may be constructed so that each $F_{t}$ has a continuous derivative on $J-K$. We have no assurance, however, that the functions $F_{t}$ will have derivatives at fixed points of $f$, even though $f$ has a derivative at these points. An interesting problem is that of determining conditions for $f$ that will guarantee that we can construct the flow $F$ in such a manner that each function $F_{t}$ has a continuous derivative on all of $J$. We obtain a few results pertaining to this problem in the next section.

3. Flows of continuously differentiable functions. Throughout this section we assume that $f$ is a function which has the following properties: 
(i) $f$ is a homeomorphism of a half open interval $(a, b]$ onto itself;

(ii) $f$ has a continuous derivative on $(a, b]$;

(iii) $f(x)>x$ for $a<x<b$;

(iv) $f^{\prime}(x)>0$ for $a<x \leqq b$;

(v) $f^{\prime}$ is monotone nonincreasing on the interval $(a, b]$.

We are going to prove that there exists a unique flow $F$ such that $F_{1}=f$ and such that $F_{t}$ has a continuous derivative on $(a, b]$ for each real number $t$. It is clear that if such a flow exists, then $f$ must commute with $F_{t}$ for each $t$. Thus, in trying to construct the flow $F$, it is reasonable to first try to determine the set of all continuously differentiable order preserving homeomorphisms that commute with $f$.

Using conditions (i)-(v) above, it is possible to prove the following lemma. Since the proof is fairly straightforward, it is omitted.

Lemma 2. If $a<x<b$ and $a<y<b$, then the infinite product

$$
\prod_{n=0}^{\infty}\left[f^{\prime}\left(f^{n}(x)\right) / f^{\prime}\left(f^{n}(y)\right)\right]
$$

converges. Moreover, if $a<a^{*}<b^{*}<b$, then for each fixed $y$, the infinite product converges uniformly in $x$ for $a^{*} \leqq x \leqq b^{*}$.

In view of the above lemma, we obtain a continuous function $\phi$ if we define $c=(a+b) / 2$ and then define $\phi(x)=\prod_{n=0}^{\infty}\left[f^{\prime}\left(f^{n}(x)\right) / f^{\prime}\left(f^{n}(c)\right)\right]$ for $a<x<b$.

TheоRем 2. If $g$ is a continuously differentiable homeomorphism of $(a, b]$ onto itself and $g$ commutes with $f$, then

$$
g^{\prime}(x)=g^{\prime}(b) \phi(x) / \phi(g(x))
$$

for $a<x<b$.

Proof. Since $f$ and $g$ commute, we obtain for all $x$,

$$
f(g(x))=g(f(x)) .
$$

We now differentiate each side of the above equation, obtaining

$$
f^{\prime}(g(x)) g^{\prime}(x)=g^{\prime}(f(x)) f^{\prime}(x) .
$$

We solve for $g^{\prime}(x)$, obtaining

$$
g^{\prime}(x)=\left[f^{\prime}(x) / f^{\prime}(g(x))\right] g^{\prime}(f(x)) .
$$

Next we replace $x$ by $f^{k}(x)$ in the above equation, and use the fact that $g$ and $f^{k}$ commute. We obtain

$$
g^{\prime}\left(f^{k}(x)\right)=\left[f^{\prime}\left(f^{k}(x)\right) / f^{\prime}\left(f^{k}(g(x))\right)\right] g^{\prime}\left(f^{k+1}(x)\right) .
$$


If $n$ is a positive integer, it follows that

$$
g^{\prime}(x)=\left\{\prod_{k=0}^{n}\left[\frac{f^{\prime}\left(f^{k}(x)\right)}{f^{\prime}\left(f^{k}(g(x))\right)}\right]\right\} g^{\prime}\left(f^{n+1}(x)\right) .
$$

Since $g^{\prime}$ is continuous at $b$ and $\lim _{n \rightarrow \infty} f^{n+1}(x)=b$, we obtain

$$
g^{\prime}(x)=\left\{\prod_{k=0}^{\infty}\left[\frac{f^{\prime}\left(f^{k}(x)\right)}{f^{\prime}\left(f^{k}(g(x))\right)}\right]\right\} g^{\prime}(b)=g^{\prime}(b) \frac{\phi(x)}{\phi(g(x))} .
$$

COROLlARY 1. Under the same hypotheses as for the above theorem, $g^{\prime}(b) \neq 0$.

It is easy to prove under the hypotheses for $f$ that $0<f^{\prime}(b)<1$. We define $A=f^{\prime}(b)$. It follows from the above results that any continuously differentiable homeomorphism on $(a, b]$ that commutes with $f$ must be a solution of a differential equation of the form

$$
d y / d x=A^{t} \phi(x) / \phi(y) .
$$

We now study the existence and uniqueness of solutions of the differential equation

$$
E(t): \quad d y / d x=A^{t} \phi(x) / \phi(y) .
$$

Lemma 3. Suppose that $t$ is a real number and that $a \leqq d<b$. Then there exists at most one continuous function $g$ on $(d, b]$ such that $g$ satisfies $E(t)$ on $(d, b)$ and $g(b)=b$.

Proof. Suppose that $g$ and $h$ are continuous on $(d, b]$, both are solutions of $E(t)$ on the interval $(d, b)$, and $g(b)=h(b)=b$. If $g$ and $h$ are not identical, then there exists a point $p$ in the interval such that $g(p) \neq h(p)$. We may assume without loss of generality that $g(p)$ $>h(p)$. There exists a point $q, p<q \leqq b$, such that $g(q)=h(q)$ and $g(x)>h(x)$ for $p \leqq x<q$. We define $w(x)=g(x)-h(x)$. The Mean Value Theorem yields a point $r, p<r<q$, for which

$$
\begin{aligned}
\frac{g(p)-h(p)}{p-q} & =\frac{w(p)-w(q)}{p-q}=w^{\prime}(\boldsymbol{r})=g^{\prime}(\boldsymbol{r})-h^{\prime}(\boldsymbol{r}) \\
& =A^{t} \phi(\boldsymbol{r})\left\{\frac{1}{\phi(g(\boldsymbol{r}))}-\frac{1}{\phi(h(\boldsymbol{r}))}\right\} .
\end{aligned}
$$

Since $g(r)>h(r)$ and $\phi$ is nonincreasing, it follows that $A^{t} \phi(r)\{1 / \phi(g(r))-1 / \phi(h(r))\}$ is nonnegative. We now have a contradiction, since $[g(p)-h(p)] /(p-q)$ is negative.

We next define a function $\Phi$ on the interval $(a, b]$ by letting $\Phi(x)=\int_{x}^{b} \phi(t) d t$. 
LEMMA 4. $\Phi$ is an order reversing homeomorphism of $(a, b]$ onto $[0, \infty)$.

Proof. It is obvious that $\Phi$ is continuous and strictly monotone decreasing, and that $\Phi(b)=0$. Hence it is sufficient to prove that $\lim _{x \rightarrow a} \Phi(x)=\infty$.

Let us assume that $a<x<c$. We first observe that

$$
\Phi(x) \geqq \int_{x}^{c} \phi(t) d t .
$$

However, for $x \leqq t \leqq c, \phi(t) \geqq \prod_{k=0}^{n}\left[f^{\prime}\left(f^{k}(t)\right) / f^{\prime}\left(f^{k}(c)\right)\right]$ for each $n$. Moreover, it is easy to prove by induction on $n$ that

$$
\prod_{k=0}^{n} f^{\prime}\left(f^{k}(t)\right)=\frac{d}{d t} f^{n+1}(t)
$$

It follows from these facts that $\phi(x) \geqq\left[f^{n+1}(c)-f^{n+1}(x)\right] / \prod_{k=0}^{n} f^{\prime}\left(f^{k}(c)\right)$ for every $n$. Now let $B$ be any positive number. It is easy to see that $\lim _{n \rightarrow \infty} f^{n+1}(c)=b$ and $\lim _{n \rightarrow \infty} \prod_{k=0}^{n} f^{\prime}\left(f^{k}(c)\right)=0$. Thus there exists an integer $m$ such that $f^{m+1}(c)>(a+2 b) / 3$ and

$$
\prod_{k=0}^{m} f^{\prime}\left(f^{k}(c)\right)<(b-a) / 3 B
$$

Now choose $\epsilon>0$ such that if $a<x<a+\epsilon$ then $f^{m+1}(x)<(2 a+b) / 3$. It now follows that if $a<x<a+\epsilon$ then $\Phi(x)>B$. Therefore,

$$
\lim _{x \rightarrow a} \Phi(x)=\infty \text {. }
$$

LEMma 5. If $g$ is an order preserving homeomorphism of $(a, b]$ onto itself and $g$ commutes with $f$, then $\lim _{x \rightarrow b} \phi(x) / \phi(g(x))=1$.

Proof. It is easy to verify that $\lim _{x \rightarrow b} \phi(x) / \phi\left(f^{m}(x)\right)=1$ if $m$ is an integer. We shall show that there exists an integer $n$ such that $f^{n-1}(x) \leqq g(x) \leqq f^{n+2}(x)$ for $a<x<b$. Since $\phi$ is monotone, our lemma will follow from the inequalities

$$
\phi(x) / \phi\left(f^{n-1}(x)\right) \leqq \phi(x) / \phi(g(x)) \leqq \phi(x) / \phi\left(f^{n+2}(x)\right) .
$$

There exists an integer $n$ such that $f^{n}(c) \leqq g(c) \leqq f^{n+1}(c)$. Now let $x$ be any member of the interval $(a, b)$. There exists an integer $k$ such that $f^{k}(c) \leqq x \leqq f^{k+1}(c)$. If we now use the fact that each power of $f$ is monotone increasing and commutes with $g$, we obtain

$f^{n-1}(x) \leqq f^{n+k}(c) \leqq g\left(f^{k}(c)\right) \leqq g(x) \leqq g\left(f^{k+1}(c)\right) \leqq f^{n+k+2}(c) \leqq f^{n+2}(x)$. 
Thus, $f^{n-1}(x) \leqq g(x) \leqq f^{n+2}(x)$, and our lemma follows.

THEOREM 3. There exists a unique flow $F$ on $(a, b]$ such that $F_{1}=f$. and such that each $F_{t}$ is continuously differentiable on $(a, b]$. Moreover, if $g$ is any continuously differentiable homeomorphism of $(a, b]$ onto itself such that $g$ commutes with $f$, then there exists a real number $t$ such that $F_{t}=g$.

Proof. We define $F_{t}(x)=\Phi^{-1}\left(A^{\iota} \Phi(x)\right)$ for $a<x \leqq b$. It is easy to verify that each function $F_{t}$ is a homeomorphism of $(a, b]$ onto itself. Moreover, it is easy to prove that $F_{s} F_{t}=F_{s+t}$ for all $s$ and $t$, and that $F$ is continuous. Thus $F$ is a flow on the interval $(a, b]$.

Since $\Phi^{\prime}(x)=-\phi(x)$, it follows that $F_{t}^{\prime}(x)=A^{\prime} \phi(x) / \phi\left(F_{t}(x)\right)$ and hence $F_{t}$ satisfies the differential equation $E(t)$ on the interval $(a, b)$. Since $f$ obviously satisfies $E(1)$, it follows from Lemma 3 that $F_{1}=f$.

Since $F_{1}=f$ and $F$ is a flow, it follows that $F_{t}$ commutes with $f$ for each $t$. We now use Lemma 5 to obtain $\lim _{x \rightarrow b} F_{t}^{\prime}(x)=A^{t}$. It follows that $F_{t}$ is differentiable at $b$, and that $F_{t}^{\prime}(b)=A^{t}$. Thus each function $F_{t}$ has a continuous derivative on $(a, b]$.

Let us now prove that the flow $F$ is unique. We observe that if $n$ is a positive integer and $g$ is a continuously differentiable homeomorphism of $(a, b]$ onto itself for which $g^{n}=f$, then

$$
f^{\prime}(x)=g^{\prime}\left(g^{n-1}(x)\right) g^{\prime}\left(g^{n-2}(x)\right) \cdots g^{\prime}(x) .
$$

If we let $x$ tend to $b$, it is easy to see that we obtain

$$
f^{\prime}(b)=\left(g^{\prime}(b)\right)^{n} .
$$

It follows that $g$ satisfies the equation $E(1 / n)$. Now let $G$ be any flow of continuously differentiable functions which satisfies $G_{1}=f$. We see that $G_{1 / n}$ and $F_{1 / n}$ both satisfy $E(1 / n)$ for each positive integer $n$ and hence $G_{1 / n}=F_{1 / n}$. It follows that $G_{r}=F_{r}$ for every rational number $r$. Since flows are continuous in both variables, $G_{t}(x)=F_{t}(x)$ for all real $t$ and all $x$ in the interval $(a, b]$. Therefore $F$ is unique.

Now suppose that $g$ is a continuously differentiable homeomorphism of $(a, b]$ onto itself and that $g$ commutes with $f$. It follows from Corollary 1 that $g^{\prime}(b) \neq 0$. Thus, since $g$ is order preserving, $g^{\prime}(b)>0$ and there exists a real number $t$ such that $A^{t}=g^{\prime}(b)$. It follows from Theorem 2 that $g$ satisfies the differential equation $E(t)$, and since $F_{t}$ also satisfies $E(t)$, Lemma 3 implies that $F_{t}=g$.

It is obvious that we may extend the domain of $f$ and of the functions $F_{t}$ to the closed interval $[a, b]$ by defining $F_{t}(a)=a$, and we shall then obtain a flow $F$ on $[a, b]$. However, as is shown by the following example, it is not necessarily true that each of the functions 
$F_{t}$ will have a derivative at $a$, even though $f$ has a derivative at $a$.

EXAMPLE. Let $g(x)=4 x /(3 x+1)$ for $0 \leqq x \leqq 1$. Next define a function $f$ such that $f(x)=2 x$ for $0 \leqq x \leqq 1 / 3 ; f(x)=g(x)$ for $1 / 2 \leqq x \leqq 1$; $f(x)$ arbitrary for $1 / 3 \leqq x \leqq 1 / 2$, subject only to the requirement that $f$ satisfy the conditions (i) $-(v)$ listed at the beginning of $\S 3$. It is easily seen that it is possible to define such a function $f$. Theorem 3 implies that there exist flows $G$ and $F$ such that $G_{1}=g, F_{1}=f$ and, all of the functions $F_{t}$ and $G_{t}$ are continuously differentiable on $(0,1]$. If one makes use of the uniqueness of $G$, it is easily verified that $G_{t}(x)=4^{t} x /\left(\left(4^{t}-1\right) x+1\right)$. Since $g(x)=f(x)$ for $1 / 2 \leqq x \leqq 1$, it is easily seen that $G_{1 / 2}$ and $F_{1 / 2}$ satisfy the same differential equation on the interval $1 / 2 \leqq x \leqq 1$. It follows from Lemma 3 that $F_{1 / 2}(x)=G_{1 / 2}(x)$ for $1 / 2 \leqq x \leqq 1$, and we use this fact to compute $F_{1 / 2}(1 / 2)=2 / 3$. Next we define points $p_{0}, p_{1}, p_{2}, \cdots$ by letting $p_{0}=1 / 2, p_{1}=1 / 3$, and $p_{n}=F_{-1}\left(p_{n-2}\right)$ for $n \geqq 2$. Since $F_{1 / 2}\left(p_{0}\right)=2 / 3=F_{1}\left(p_{1}\right)$, we obtain $F_{1 / 2}\left(p_{1}\right)=p_{0}$. It is easy to prove by induction that $F_{1 / 2}\left(p_{n}\right)=p_{n-1}$ for every positive integer $n$. Since $F_{-1}(x)=x / 2$ for $0 \leqq x \leqq 1 / 2$, it is easy to compute the values of the numbers $p_{n}$, and to verify that the difference quotient $\left(F_{1 / 2}\left(p_{n}\right)-F_{1 / 2}(0)\right) /\left(p_{n}-0\right)$ is equal to $3 / 2$ if $n$ is odd and is equal to $4 / 3$ if $n$ is even. Thus $F_{1 / 2}$ does not have a derivative at 0 .

This example demonstrates that it is possible to have a function $f$ which is continuously differentiable on the closed interval $[a, b]$ and which satisfies conditions (i)-(v) on the half-open interval $(a, b]$, but which cannot be embedded in a flow of functions that are continuously differentiable on the closed interval $[a, b]$.

We do, however, have the following result.

THEOREM 4. If $f$ is continuously differentiable over the closed interval $[a, b]$ and satisfies (i)-(v) on $(a, b]$, then the set $G$ of all continuously differentiable order preserving homeomorphisms $g$ of $[a, b]$ onto itself for which $g$ commutes with f forms a group which is isomorphic to either the group of real numbers or the group of integers.

Proof. We use Theorem 3 to obtain a flow $F$ on $[a, b]$ such that each of the functions $F_{t}$ has a continuous derivative on $(a, b]$, and $F_{1}=f$. It follows from Theorem 3 that if $g \in G$, then there exists a real number $t$ such that $F_{t}=g$. We let $S$ be the set of all real numbers $t$ for which $F_{t} \in G$. If we use the fact that $F_{t+s}^{\prime}(x)=F_{t}^{\prime}\left(F_{s}(x)\right) F_{s}^{\prime}(x)$ for $a<x<b$, it is easy to see that if $t$ and $s$ are members of $S$ then $t+s$ is also a member of $S$. Moreover, if $t$ is in $S$ then $-t$ is in $S$. Thus $S$ is a subgroup of the reals. It is not difficult to prove that $G$ is a group which is isomorphic to $S$, and consequently our theorem is 
proved if we can show that either $S$ is the set $R$ of all real numbers or else $S$ is a nondegenerate discrete subgroup of $R$.

We observe that $S$ is nondegenerate since $1 \in S$. Now assume that $S$ is isomorphic to neither $R$ nor the group of integers. It then follows that both $S$ and $R-S$ are dense in $R$, and this implies that $R-S$ is uncountable. We shall now show that this is impossible. In order to accomplish this, we define $u_{t}$ to be the lower derivate of $F_{t}$ at $a$, and we define $v_{t}$ to be the upper derivate of $F_{t}$ at $a$. It is easily seen that if $s<t$, then $F_{s}(x)<F_{t}(x)$ for $a<x<b$, and it follows that if $s<t$ then $u_{s} \leqq u_{t}$ and $v_{s} \leqq v_{t}$. Now let $r$ and $t$ be members of $R-S, r<t$. Since $S$ is dense in $R$, there exists $s \in S$ such that $r<s<t$. We see that $u_{r}<v_{r} \leqq v_{s}=u_{s} \leqq u_{t}<v_{t}$. Thus, by defining $L(t)$ to be the open interval $\left(u_{t}, v_{t}\right)$ for each $t \in R-S$, we obtain a one-to-one function $L$ that maps $R-S$ onto a set of mutually exclusive open intervals. This implies that $R-S$ is countable, and we have a contradiction.

We conclude by listing two unsolved problems that are of interest.

PROBLEM 1. Find conditions on $f$ that are necessary and sufficient for $S=R$, where $S$ and $R$ are the sets defined in the proof of Theorem 4 .

PRoBlem 2. Replace condition ( $\mathrm{v}$ ) on $f$ by a weaker condition.

The solution of these problems would constitute a major step toward obtaining necessary and sufficient conditions that it be possible to embed a continuously differentiable homeomorphism (having arbitrarily many fixed points) of an interval onto itself in a flow of continuously differentiable homeomorphisms.

\section{BIBLIOGRAPHY}

1. A. G. Walker, Commutative functions (I), Quart. J. Math. Oxford Ser. vol. 17 (1946) pp. 65-92.

2. G. J. Whitrow, On equivalent observers, Quart. J. Math. Oxford Ser. vol. 6 (1935) pp. 249-260.

The University of Georgia 